\title{
Blessures associées à des produits de consommation au Canada : revue systématique de la littérature
}

\author{
S. A. Huchcroft, Ph. D. (1); C. R. McGowan, Ph. D. (2); F. Mo, Ph. D. (3)
}

Cet article a fait l'objet d'une évaluation par les pairs.

\section{Résumé}

Objectifs: Effectuer une revue systématique de la littérature portant sur les blessures associées à certains produits de consommation.

Méthodologie: Nous avons analysé 46 rapports de recherche de nature empirique et 32 rapports de surveillance du Système canadien hospitalier d'information et de recherche en prévention des traumatismes (SCHIRPT) dans le but de déterminer le nombre approximatif de blessures par année associées à chaque produit ainsi que toute tendance dans les fréquences. Nous avons également extrait des rapports les pourcentages des blessures qui ont donné lieu à une hospitalisation, qui semblaient résulter du produit lui-même et qui étaient associées à une utilisation inappropriée ou dangereuse ou à l'absence de casque.

Résultats: Les équipements de sport et de jeu de plein air semblent être associés aux nombres de blessures les plus élevés. Une proportion relativement importante de blessures semble résulter d'une utilisation inappropriée ou dangereuse du produit et du non-respect des mesures de sécurité requises.

Conclusion: Cette revue a permis de relever plusieurs sujets de préoccupation à propos des blessures associées à des produits de consommation : absence de casque lors de l'utilisation de patins à roues alignées, de traîneau, de planche à neige, de skis alpins ou de motomarine; conduite d'un véhicule tout-terrain (VTT) ou d'une motoneige avec facultés affaiblies par l'alcool; conduite d'une motoneige à une vitesse excessive; conception défectueuse de certains équipements de terrain de jeu; entreposage et utilisation non sécuritaires d'allumettes.

\section{Introduction}

La sécurité des produits de consommation est considérée comme une question prioritaire en matière de prévention des blessures au Canada ${ }^{1}$. D’après les données du Système canadien hospitalier d'information et de recherche en prévention des traumatismes (SCHIRPT) ${ }^{*}, 2$, près de la moitié des blessures subies par les enfants et les jeunes (âgés de 19 ans et moins) sont liées à des produits de consommation ${ }^{3}$.
$\mathrm{Au}$ moins quatre catégories générales de facteurs exercent une influence sur les blessures associées aux produits de consommation :

(1) La qualité du produit et la probabilité qu'il puisse causer des blessures en raison de ses défauts ou de ses caractéristiques, notamment bords tranchants, vice de fabrication, assemblage inadéquat ou défaillance du produit;
(2) Les facteurs liés à la manière dont le produit est utilisé, par exemple à une vitesse ou avec une force excessives, à une fin inappropriée ou de manière négligente;

(3) Le rôle du hasard, qui fait que même si un produit de bonne qualité est utilisé de manière appropriée, un accident est susceptible de survenir sans avoir pu être anticipé ou évité;

(4) L'absence, dans certains cas, d'équipement de protection permettant de prévenir les blessures ou d'en atténuer la gravité.

Dans cet article, nous présentons les résultats d'une recension systématique de la littérature portant sur les blessures liées aux produits de consommation au Canada. Nous y examinons, produit par produit, la proportion de blessures pouvant avoir été causées par un défaut du produit lui-même ou par une utilisation inappropriée ou dangereuse de celui-ci, la proportion de personnes blessées qui ne portaient pas de casque (le cas échéant) lorsqu'elles se sont blessées, la fréquence et la gravité des blessures par produit et toute tendance décelable en matière de fréquence.

\section{Méthodologie}

Définitions de "produit de consommation » et de «blessure»

Aux termes de la Loi canadienne sur la sécurité des produits de consommation (LCSPC), la définition du produit de consommation est la suivante :

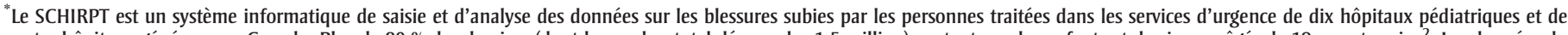

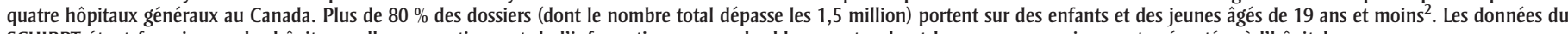
SCHIRPT étant fournies par les hôpitaux, elles ne contiennent de l'information que sur les blessures touchant les personnes qui se sont présentées à l'hôpital.

Rattachement des auteurs :

1. Consultante en épidémiologie, Calgary (Alberta), Canada

2. Department of Public Health and Policy, London School of Hygiene \& Tropical Medicine, Londres, Royaume-Uni

3. Division de l'intégration scientifique, Centre de prévention et de contrôle des maladies chroniques, Agence de la santé publique du Canada, Ottawa (Ontario), Canada

Correspondance : Catherine R. McGowan, Faculty of Public Health \& Policy, London School of Hygiene \& Tropical Medicine, 15-17 Tavistock Place, Londres WC1H 9SH, Royaume-Uni; tél. : (011 44) 207927 2888; courriel : Catherine.McGowan@Ishtm.ac.uk 
« Produit - y compris tout composant, partie ou accessoire de celui-ci - dont on peut raisonnablement s'attendre à ce qu'un individu l'obtienne en vue d'une utilisation à des fins non commerciales, notamment à des fins domestiques, récréatives ou sportives. Est assimilé à un tel produit son emballage. 4 , article 2

Sont exclus de cette définition les armes à feu, les munitions et les explosifs, les arbalètes, les aliments et drogues, les produits antiparasitaires, les aliments pour animaux, les engrais, les végétaux, les semences, les substances réglementées, les produits aéronautiques, les animaux, le tabac et les produits du tabac, les bateaux et les véhicules au sens de l'article 2 de la Loi sur la sécurité automobile (LSA) (c'est-à-dire de tout "véhicule automobile, ou faisant partie d'un attelage automobile, qui peut circuler sur la route [...] » ${ }^{5}$, article 2 ). Toutefois, même s'ils relèvent en principe de la LSA, les véhicules motorisés récréatifs tout-terrain tels que les véhicules toutterrain (VTT), les motoneiges et les trottinettes motorisées ont été inclus dans cette étude. Les blessures subies lors de la pratique de sports d'équipe faisant appel à de l'équipement comme le soccer, le hockey ou le baseball ont été exclues de l'étude, car elles appartiennent à des catégories relativement discrètes qui justifieraient une analyse distincte, tout comme les lésions découlant d'un empoisonnement. Enfin, nous avons inclus les équipements de terrain de jeu, car, même s'ils ne sont pas à strictement parler de nature domestique, ils sont inclus dans les produits de consommation de Santé Canada ${ }^{6}$.

La définition du terme "blessure » a été difficile, car elle ne figure pas dans la LCSPC. Toutefois, dans le contexte de cette étude, une blessure sous-entend l'attribution d'un code de la Classification statistique internationale des maladies et des problèmes de santé connexe, $10^{e}$ révision (CIM$10)^{7}$ correspondant à une cause externe de blessure non intentionnelle (V01-X59 et Y85-Y86), à l'exclusion des blessures lors d'un accident de la circulation routière survenu sur une voie ou une rue publique où la personne blessée était dans le véhicule, ainsi que des cas d'empoisonnement, de complications suite à des soins médicaux et chirurgicaux, de blessure auto-infligée, d'agression, d'intention indéterminée, d'intervention légale ou de guerre. Sont incluses les lésions traumatiques de la tête, du cou, du thorax, de l'abdomen, des lombes, du rachis lombaire, du bassin, de l'épaule et de la partie supérieure du bras, du coude et de l'avant-bras, du poignet et de la main, de la hanche et de la cuisse, du genou et de la jambe, de la cheville et du pied (codes CIM-10 de région S00-S99), ainsi que les lésions traumatiques de plusieurs parties du corps, de siège non précisé du tronc, d'un membre ou d'une région du corps, les effets dus à un corps étranger ayant pénétré dans un orifice naturel, ainsi que les brûlures et les corrosions (codes CIM-10 T00-T32). Compte tenu de la rareté des articles scientifiques donnant une définition du terme «blessure » ou indiquant des codes de la CIM, notre revue systématique de la littérature s'est appuyée sur la définition de la blessure donnée par les auteurs des documents analysés. Toutes les blessures ont été soignées par un professionnel de la santé, le plus souvent au service des urgences d'un hôpital.

\section{Stratégie de recherche utilisée dans la revue systématique de la littérature}

Nous avons effectué des recherches dans sept bases de données couvrant le secteur paramédical, la médecine clinique, les soins infirmiers et les politiques de santé (CINAHL Plus, EMBASE, MEDLINE), les sciences générales (Web of Science), la santé publique (Global Health), les sciences sociales (Social Policy \& Practice) et les sciences de la vie appliquées (CAB Abstracts). La recherche a été effectuée en février 2011 et a couvert la littérature publiée au cours des deux décennies précédentes.

Les termes de recherche étaient les suivants : Canad $^{*}+$ (injur $^{*}$ ou accident*) + (consumer* ou product*). À titre de contre-vérification rapide de l'exhaustivité de cette recherche, nous avons examiné les résultats, beaucoup plus nombreux, obtenus lors d'une recherche antérieure pour un autre projet portant sur les facteurs de risque liés aux blessures et réalisé en juillet 2010. Les termes de recherche des facteurs de risque étaient Canad* (injur* $^{*}$ ou accident* ${ }^{*}+$ (risk factor ou inciden* ou caus* $^{*}$ ou hospital* ou mortality ou disabilit* ou fall*). Cette recherche de portée plus large n'a permis de recenser aucune autre étude sur les blessures associées à des produits de consommation.

Nous avons également pris en considération plusieurs autres sources, notamment des livres, des chapitres de livre, des articles électroniques et des rapports. Trois documents publiés par l'Agence de la santé publique du Canada (ASPC) ont constitué des sources importantes : Étude des blessures chez les enfants et les jeunes, Édition 2009 - Pleins feux sur la sécurité des produits de consommation ${ }^{3}$, SCHIRPT - Rapports de blessures et échantillons de données ${ }^{8}$ et Inventaire des sources de données et des activités de surveillance des blessures ${ }^{9}$. Nous avons analysé les bibliographies correspondantes afin de repérer des articles pertinents qui n'auraient pas été recensés par notre stratégie de recherche. Nous avons pris en considération les articles publiés dans l'une ou l'autre des langues officielles.

\section{Critères d'inclusion et d'exclusion}

Nous avons inclus les rapports des recherches de nature empirique publiés au Canada portant sur les blessures associées à des produits de consommation et dans lesquels étaient présentées des estimations chiffrées importantes, mais nous avons exclu les commentaires et les éditoriaux. Nous avons utilisé les comptesrendus de lecture comme source pour des références bibliographiques qui nous auraient échappé lors de la recherche initiale, sans toutefois les inclure dans le matériel empirique lui-même.

\section{Indices pour la détermination des priorités en matière de gestion des risques}

Après avoir analysé toutes les études recensées, nous avons choisi six indices pour faciliter la détermination des priorités en matière de réduction des blessures : (1) le nombre annuel approximatif de blessures (estimé en divisant le nombre de blessures déclarées par la durée de la période couverte); (2) la gravité relative de la blessure exprimée en pourcentage des personnes blessées admises à l'hôpital; 
(3) les tendances décelables; (4) la proportion des blessures causées par le produit lui-même (p. ex. défauts); (5) la proportion des blessures causées par une utilisation inappropriée ou risquée (le SCHIRPT consigne les circonstances entourant la survenue de la blessure, p. ex. vitesse excessive ou, dans le cas des lits superposés, saut à partir du lit du haut); (6) la proportion des personnes blessées qui ne portaient pas de casque au moment de la survenue de la blessure (dans le cas où le port d'un casque était jugé approprié et où cette information a été consignée). Nous avons sélectionné comme produits de consommation devant être considérés comme prioritaires ceux qui étaient associés à environ 500 blessures ou plus par an, à $20 \%$ ou plus de personnes blessées qui ont été admises à l'hôpital, à une tendance à la hausse du nombre de blessures, à $5 \%$ ou plus de blessures imputables au produit lui-même, à $50 \%$ ou plus de personnes blessées ayant utilisé le produit de façon inappropriée ou négligente, et à $50 \%$ ou plus de personnes qui ne portaient pas de casque au moment où elles se sont blessées. Les seuils des indices ont été choisis arbitrairement pour pouvoir répertorier entre cinq et dix produits de consommation chacun. Les produits de consommation pour lesquels les blessures étaient soit les plus nombreuses, soit les plus graves et qui satisfaisaient à au moins un des quatre autres critères ont été considérés comme prioritaires en matière de gestion des risques.

\section{Résultats}

La consultation des bases de données a permis de repérer 703 enregistrements après suppression des doublons, dont 46 qui satisfaisaient à nos critères d'inclusion concernant les recherches empiriques quantitatives originales (tableau 1). En respectant les contraintes en matière de temps et de ressources de ce projet, nous avons obtenu le texte intégral des articles correspondant à 37 de ces enregistrements. Pour les 9 autres, nous avons utilisé l'information présentée dans le résumé. Nous avons inclus les articles publiés uniquement sous forme de résumé afin d'éviter tout biais associé à l'utilisation des seules références pour lesquelles l'article intégral avait pu être obtenu, ainsi que pour pouvoir effectuer d'autres tenta- tives en vue de trouver ces textes. De plus, 32 rapports en ligne du SCHIRPT trouvés grâce à une recherche sur Internet en utilisant les mêmes mots clés sont venus enrichir les données issues des bases de données documentaires.

Le tableau 1 résume les résultats de la revue systématique de la littérature par produit de consommation en ce qui concerne le nombre annuel de blessures, le pourcentage des personnes blessées admises à l'hôpital, le type et le siège les plus courants de la blessure et toute tendance relevée. Les résultats sont présentés par groupe d'âge le plus touché, du plus jeune au plus âgé.

D’après les publications recensées, dans lesquelles prédominent les blessures subies par les enfants et les adolescents, les équipements de sport et de jeu de plein air semblent être associés aux nombres de blessures les plus élevés. Les équipements de terrain de jeu (p. ex. balançoires, portiques d'escalade, glissoires, balançoires à bascule) comme les bicyclettes sont associés à plus de 3000 blessures. Viennent ensuite les activités hivernales de descente à l'aide d'un traîneau, d'une planche à neige ou de skis, avec environ 1000 blessures chaque année par catégorie. Parmi les autres produits de consommation associés à 500 à 1000 blessures par an figurent les planches à roulettes, les patins à roues alignées, les patins à glace et les trampolines.

Les produits de consommation utilisés dans la maison jouent un rôle plus important en termes de gravité que de nombre absolu de blessures. La gravité des blessures est définie ici par la proportion de personnes blessées qui ont été hospitalisées. Si le taux d'hospitalisation peut servir de variable de substitution pour comparer la gravité des blessures d'un produit de consommation à l'autre, le fait que les données du SCHIRPT n'incluent pas d'information sur les personnes décédées avant d'avoir été transportées à l'hôpital entraîne probablement une sousestimation de la gravité de certains types de blessures associées aux produits de consommation. Plus de la moitié des enfants blessés par un cordon de store ou de rideau ou dans une piscine sont admis à l'hôpital, tout comme $25 \%$ à $30 \%$ des enfants ayant subi une brûlure à cause d'un foyer au gaz ou d'allumettes et plus de $20 \%$ des personnes blessées par une tondeuse à gazon. À l'extérieur de la maison, les produits de consommation les plus susceptibles d'être associés à des blessures graves sont les véhicules récréatifs motorisés comme les VTT et les motoneiges (plus de $30 \%$ des blessés admis à l'hôpital), les motomarines et les motos tout-terrain (plus de $20 \%$ des blessés admis à l'hôpital).

Les produits de consommation pour lesquels le nombre de blessures pourrait être en hausse sont les sièges de bain pour bébés, les trampolines, les chaussures à roulettes, les trottinettes motorisées, les VTT, les motos tout-terrain, les planches à neige, les tubes aquatiques et les aimants.

Les tableaux 2 à 4 indiquent les circonstances liées aux blessures. Comme l'essentiel de la recherche décrivant les circonstances de survenue de la blessure s'appuie sur les données du SCHIRPT, les produits de consommation utilisés par les enfants et les jeunes prédominent. D’après les données disponibles, il semble que les blessures associées aux caractéristiques des produits soient relativement rares (tableau 2). Les balançoires pour bébés semblent être le produit le plus souvent en cause avec $12,0 \%$ des blessures imputables au produit lui-même, suivies des chaussures à roulettes $(7,6 \%)$, des trottinettes motorisées (7,5\%), de l'équipement de terrain de jeu (6,6\%), des lits d'enfants, berceaux et bassinettes (5,7 \%), des parcs pour enfants $(3,8 \%)$, des barrières pour enfants $(3,4 \%)$, des véhicules-jouets motorisés (3,0\%), des lits superposés $(1,4 \%)$ et des trottinettes non motorisées (1,0\%).

Une proportion relativement élevée des blessures est attribuable à une utilisation inappropriée ou dangereuse d'un produit (tableau 3). Dans le cas des aimants, presque toutes les blessures (93,9 \%) semblent être le résultat d'une utilisation inappropriée ou dangereuse (p. ex. ingestion ou insertion dans le nez). Environ les trois quarts des blessures survenues lors d'un accident de motoneige sont attribuables aux facultés affaiblies par l'alcool ou à une 
TABLEAU 1

Principales caractéristiques des blessures associées aux produits de consommation

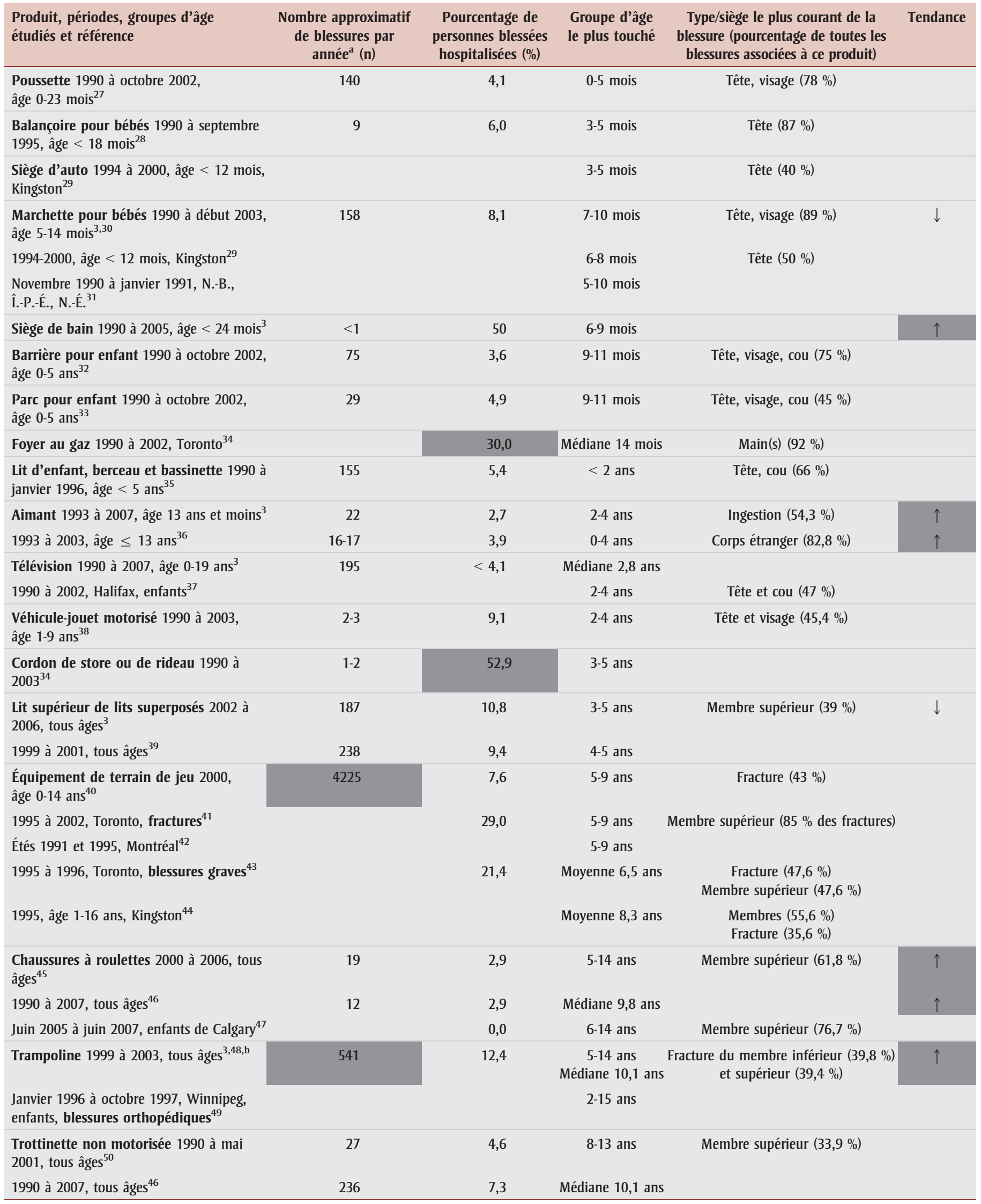


TABLEAU 1 (Suite)

Principales caractéristiques des blessures associées aux produits de consommation

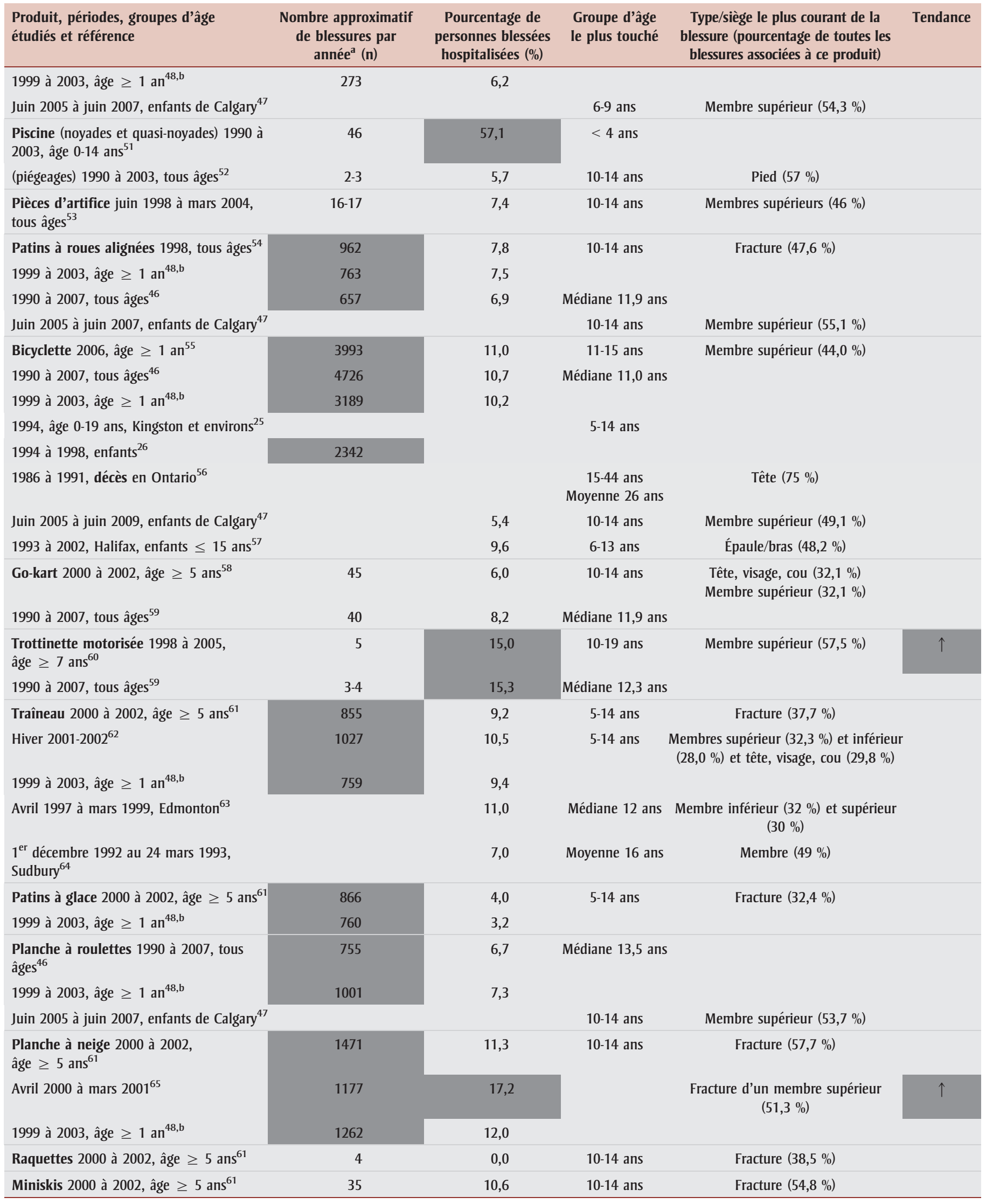


TABLEAU 1 (Suite)

Principales caractéristiques des blessures associées aux produits de consommation

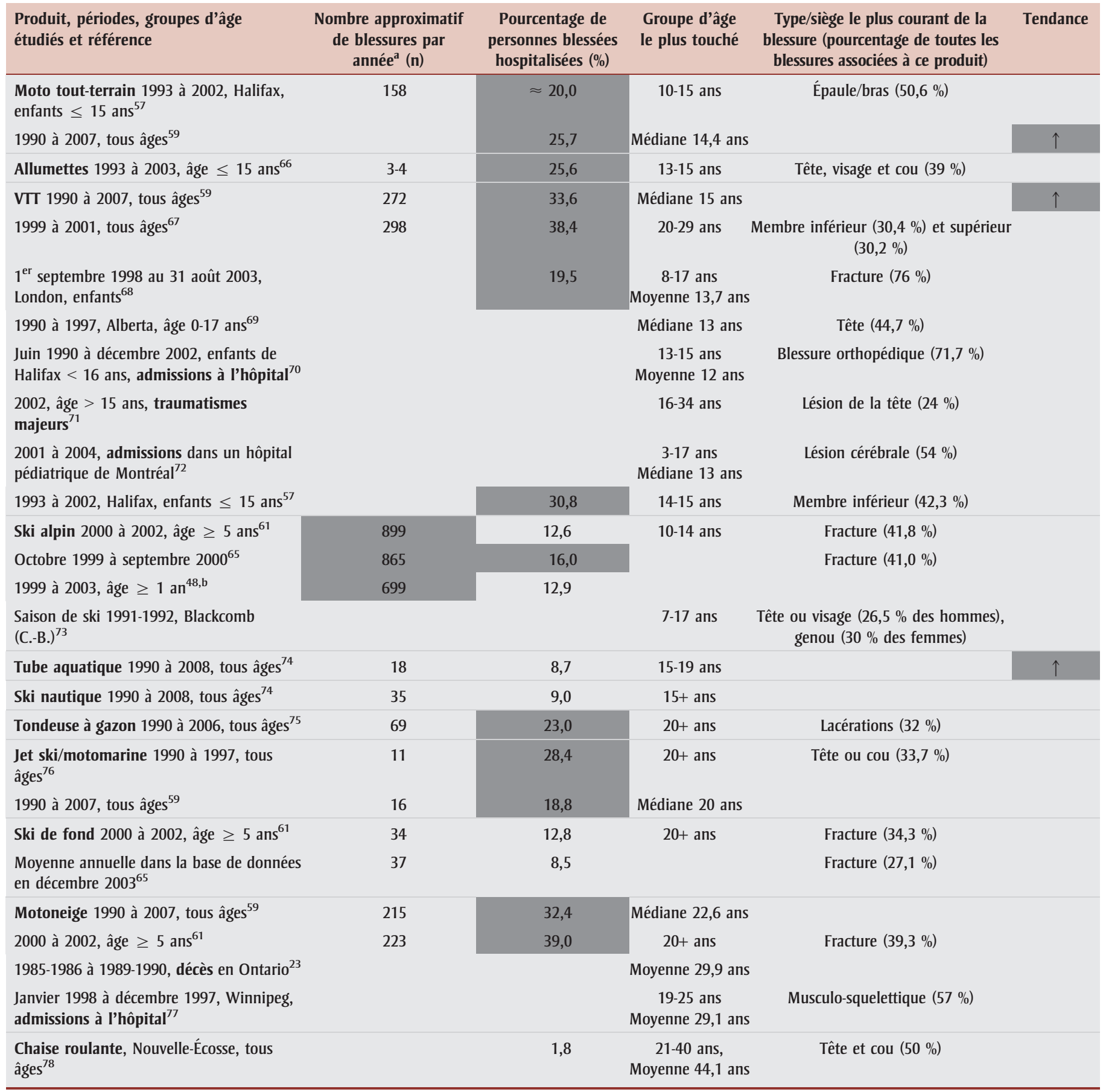

Abréviations : C.-B., Colombie-Britannique; î.-P.-E., île-du-Prince-Édouard; N.-B., Nouveau-Brunswick; N.É., Nouvelle-Écosse; SCHIRPT, Système canadien hospitalier d'information et de recherche en prévention des traumatismes; VTT, véhicule tout-terrain.

Remarque : Les éléments ombrés sont associés à au moins 500 blessures par an, à des taux d'hospitalisation de $20 \%$ ou plus ou à une tendance à la hausse du nombre de blessures.

a Données du SCHIRPT.

b L'article sur les trampolines inclut une comparaison avec d'autres équipements de nature récréative.

vitesse excessive. Les facultés affaiblies par l'alcool sont en cause dans un peu plus de la moitié des blessures associées aux VTT. Plus de $70 \%$ des blessures liées aux allumettes découlent de leur utilisation inappropriée (p. ex. jeu ou ingestion). De même, le fait de laisser seul un enfant dans un siège de bain est la raison la plus courante de blessure dans cette catégorie, avec $60 \%$ des blessures liées à ce produit.
Parmi les autres causes de blessures, on retrouve le fait de laisser une barrière pour enfants ouverte ou mal fermée $(43,4 \%$ des blessures), de jouer, de sauter, de se tenir debout sur le lit supérieur de lits super- 
TABLEAU 2

Proportion des blessures causées par des caractéristiques du produit

\begin{tabular}{lccc} 
Produit & $\begin{array}{c}\text { Pourcentage des } \\
\text { blessures causées par } \\
\text { un défaut du produit \% }\end{array}$ & $\begin{array}{c}\text { Pourcentage des } \\
\text { blessures causées par } \\
\text { le piégeage du corps \% }\end{array}$ & $\begin{array}{c}\text { Pourcentage total des } \\
\text { blessures imputables } \\
\text { aux caractéristiques du } \\
\text { produit \% }\end{array}$ \\
\hline $\begin{array}{l}\text { Balançoire pour bébés } \\
\text { Chaussures à roulettes }\end{array}$ & $12,0^{28}$ & 12,0 \\
Trottinette motorisée & $7,6^{45}$ & & 7,6 \\
Équipement de terrain de jeu & $7,5^{60}$ & \multirow{2}{*}{$44^{44}$} & 7,5 \\
Lit d'enfant, berceau et & $2,2^{44, a}$ & $4,4^{35}$ & 6,6 \\
bassinette & $1,3^{35, \mathrm{~b}}$ & & 5,7 \\
Parc pour enfant & $2,2^{33}$ & $1,6^{33}$ & 3,8 \\
Barrière pour enfant & $1,2^{32}$ & $2,2^{32}$ & 3,4 \\
Véhicule-jouet motorisé & $3,0^{38}$ & & 3,0 \\
Lits superposés & $1,2^{39}$ & $0,2^{3}$ & 1,4 \\
Trottinette non motorisée & $1,0^{50}$ & & 1,0 \\
Patins à roues alignées & $0,5^{54}$ & & 0,5 \\
Bicyclette & $0,3^{55}$ & & 0,3 \\
Trampoline & $0,1^{79}$ & & 0,1 \\
\hline
\end{tabular}

a Bords rugueux.

b Dont $0.3 \%$ imputable au fait que l'enfant a mangé le bois ou a été blessé par des éclats de bois.

posés ou d'en être poussé (40,5\%), et l'absence de barrière en haut des escaliers lorsqu'un bébé se déplace en marchette $(24,7 \%)$.
Le seul type d'équipement protecteur pour lequel on disposait de données concernant plusieurs produits était le port du casque. Dans presque tous les cas (90 \%) de blessures associées aux traîneaux, aux chaussures à roulettes ou aux motoma-

TABLEAU 3

Proportion des blessures causées par l'utilisation inappropriée ou dangereuse d'un produit

\begin{tabular}{|c|c|c|}
\hline Produit & $\begin{array}{l}\text { Types d'utilisation inappropriée } \\
\text { ou dangereuse }\end{array}$ & $\begin{array}{l}\text { Pourcentage des blessures } \\
\text { causées par une utilisation } \\
\text { inappropriée ou dangereuse, \% }\end{array}$ \\
\hline Aimant & $\begin{array}{l}\text { Ingérer un aimant ou l'insérer dans le } \\
\text { nez ou l'oreille }\end{array}$ & $93,9^{3}$ \\
\hline \multirow[t]{2}{*}{ Motoneige } & Facultés affaiblies & $69,0^{23, a} ; 70,0$ à $88,0^{77}$ \\
\hline & Vitesse excessive & $82,0^{77}$ \\
\hline Allumettes & $\begin{array}{l}\text { Jouer avec des allumettes ou en } \\
\text { ingérer }\end{array}$ & $71,8^{66}$ \\
\hline Siège de bain & Laisser l'enfant seul sur son siège & $60,0^{3}$ \\
\hline Véhicule tout-terrain & Facultés affaiblies & $\leq 56,0^{71, b}$ \\
\hline Barrière pour enfant & $\begin{array}{l}\text { Barrière laissée ouverte ou mal } \\
\text { fermée }\end{array}$ & $43,4^{32}$ \\
\hline Lits superposés (lit supérieur) & $\begin{array}{l}\text { Jouer, sauter, se tenir debout sur le } \\
\text { lit, être poussé du lit }\end{array}$ & $40,5^{3}$ \\
\hline Marchette pour bébés & $\begin{array}{l}\text { Absence de barrière en haut des } \\
\text { escaliers }\end{array}$ & $24,7^{30}$ \\
\hline Bicyclette & $\begin{array}{l}\text { Facultés affaiblies ou utilisation inap- } \\
\text { propriée }\end{array}$ & $1,8^{55} ; 7,0^{56}$ \\
\hline
\end{tabular}

\footnotetext{
a Décès.

b Traumatisme majeur.
}

rines, les personnes en cause ne portaient pas de casque au moment où elles se sont blessées (tableau 4). Entre environ la moitié et les trois quarts des personnes blessées alors qu'elles faisaient du ski alpin, de la planche à neige, de la trottinette non motorisée ou du patin à roues alignées ne portaient pas de casque. De même, une proportion considérable des personnes blessées alors qu'elles conduisaient un véhicule récréatif motorisé ne portaient pas de casque. En ce qui concerne les bicyclettes et les VTT, les pourcentages de personnes blessées qui ne portaient pas de casque sont disponibles par degré de gravité de la blessure. Dans presque tous les cas de décès dans un accident de bicyclette, la personne ne portait pas de casque et, pour les blessures associées aux VTT, l'absence de casque était plus fréquente dans les cas d'hospitalisation ou de traumatisme majeur.

Aucun des 41 produits répertoriés dans le tableau 1 n'a été associé à tous les indices de priorité, mais 26 produits l'ont été pour au moins l'un d'entre eux (tableau 5). Dans l'hypothèse d'un intérêt prioritaire envers les produits associés aux blessures soit les plus fréquentes, soit les plus graves, nous avons recensé dans ce tableau les 18 produits qui se retrouvent dans l'une ou l'autre de ces deux catégories. Les 9 produits associés aux blessures les plus fréquentes diffèrent des 9 produits ayant donné lieu à une proportion plus élevée d'hospitalisation. Une sélection plus stricte des produits en utilisant au moins un autre indice réduit ce nombre à $11: 6$ qui donnent lieu à un nombre élevé de blessures et 5 qui semblent donner lieu à des blessures plus graves nécessitant une hospitalisation.

Le tableau 6 répertorie les produits de consommation et les sujets de préoccupation. Des six produits ayant donné lieu à un nombre élevé de blessures, quatre - patins à roues alignées, traîneaux, planches à neige et skis de descente - sont associés à une proportion relativement faible de cas où la personne portait un casque.

\section{Analyse}

Les revues systématiques de la littérature sont sujettes à la fois au biais de déclaration 
TABLEAU 4

Absence de casque chez les personnes blessées, par produit

\begin{tabular}{|c|c|}
\hline Produit de consommation & $\begin{array}{c}\% \text { des blessures survenues alors que l'utilisateur } \\
\text { ne portait pas de casque }\end{array}$ \\
\hline Traîneau & $99,0 \%{ }^{64} ; 93 \%{ }^{63}$ \\
\hline Chaussures à roulettes & $95,4^{47} ; 87,3^{46}$ \\
\hline Jet ski/motomarine & $90,9^{59}$ \\
\hline Skis et planche à neige & $79,1 \%{ }^{80}$ \\
\hline Planche à roulettes & $73,7^{47} ; 67,9^{46}$ \\
\hline Trottinette non motorisée & $72,4^{50} ; 66,3^{47} ; 57,6^{46}$ \\
\hline Patins à roues alignées & $56,8^{47} ; 50,5^{54} ; 49,6^{46}$ \\
\hline Motoneige & $43,1^{59}$ \\
\hline Go-kart & $39,6^{58} ; 17,8^{59}$ \\
\hline Trottinette motorisée & $38,9^{59}$ \\
\hline Bicyclette & $37,3^{55} ; 29,8^{46} ; 15,4^{47} ; 96,0^{56, a}$ \\
\hline Véhicule tout-terrain & $29,0^{59} ; 28,2^{67} ; 35,0^{68, \mathrm{~b}} ; 84^{71, \mathrm{~b}} ; 84^{72, \mathrm{c}} ; 31,7^{70, \mathrm{c}}$ \\
\hline Moto tout-terrain & $12,7^{59}$ \\
\hline
\end{tabular}

(c'est-à-dire la tendance à déclarer plutôt les résultats statistiquement significatifs que ceux ne montrant aucune association) et au biais de publication (c'est-à-dire la probabilité plus élevée de publication des rapports mettant en évidence une signification statistique). Dans cette revue, nous avons tenté de tenir compte de ces sources de biais en incluant des données non publiées et des articles dont seul le résumé avait été localisé. Une autre source possible de biais est le choix des termes de recherche. Même si tout a été mis en œuvre pour rendre cette revue la plus complète possible compte tenu de nos ressources, nous reconnaissons que l'approche aurait été plus exhaustive en effectuant une recherche par produit de consommation (avec parfois plusieurs noms différents pour le même produit, comme jetski ou motomarine) plutôt qu'en utilisant le terme générique "produit de consommation ", qui n'était pas nécessairement un mot clé dans toutes les publications.

Nous avons pris en considération une partie de la " littérature grise » (c'est-àdire celle qui ne figure pas dans les bases de données interrogées) en incluant des publications en ligne (toutes s'appuyant sur les données du SCHIRPT); toutefois, comme nous n'avons pas recherché les publications gouvernementales dans cha- cune des provinces canadiennes, certains documents pertinents nous ont peut-être échappé ${ }^{10-14}$. Nous avons supposé que les rapports nationaux utilisant les données du SCHIRPT incluaient les données utilisées dans les rapports provinciaux.

Le thème de l'association entre blessure et produit de consommation ne se prête pas facilement à l'exercice classique de revue systématique de la littérature, et ce, pour au moins deux raisons. En premier lieu, il faut souvent plusieurs années pour élaborer et réaliser une étude épidémiologique, puis pour présenter ses résultats sous forme d'article et publier celui-ci dans une revue scientifique; par conséquent, les rapports épidémiologiques publiés ne sont probablement pas représentatifs des produits ayant fait l'objet d'un rappel rapide après un rapport d'incident. En second lieu, une revue de la littérature qui couvre plusieurs années peut manquer de pertinence par rapport aux dangers actuels, car les produits dangereux peuvent avoir déjà fait l'objet d'un rappel et ne plus être disponibles, alors que de nouveaux produits et modèles ont fait leur apparition. Ainsi, les résultats de la présente recherche n'incluent pas les effets nocifs potentiels des appareils électroniques personnels, comme la perte auditive associée aux lecteurs MP3 $3^{15}$.
Compte tenu du fait que l'essentiel des données du SCHIRPT provient d'hôpitaux situés dans des centres urbains, et pour la plupart de leurs services de pédiatrie, les blessures subies par les adolescents plus âgés et par les adultes, traitées dans les hôpitaux généraux, et par les Premières Nations, les Métis, les Inuits et le reste de la population vivant dans des régions rurales ou éloignées sont sous-représentées dans cette base de données. De surcroît, dans certaines provinces et dans les territoires, aucun hôpital ne participe au SCHIRPT (Saskatchewan, Nouveau-Brunswick, Îledu-Prince-Édouard, Yukon, Territoires du Nord-Ouest et Nunavut). De plus, les lésions fatales sont sous-représentées, car le SCHIRPT ne saisit pas les données concernant les personnes décédées avant d'avoir pu être transportées à l'hôpital ${ }^{2}$. Les études dans lesquelles a été évaluée la représentativité des données du SCHIRPT ont révélé d'autres biais. Ainsi, la sensibilité variait de $30 \%$ à $91 \%$ selon les hôpitaux ${ }^{16}$; les blessures chez les enfants plus âgés étaient plus susceptibles de ne pas être comptabilisées dans certains hôpitaux ${ }^{17,18}$ mais pas dans d'autres ${ }^{16}$, tandis que les blessures plus graves (p. ex. les cas d'hospitalisation) étaient moins susceptibles de ne pas être comptabilisées dans certains hôpitaux ${ }^{17}$ et plus susceptibles de ne pas l'être dans d'autres ${ }^{16}$. Toutefois, nous avons conclu que les données recueillies étaient fiables et valides ${ }^{19}$.

Il serait intéressant que les études à venir sur les produits de consommation et les blessures au Canada se penchent sur les aspects suivants.

\section{Fraction étiologique du risque}

Une approche possible serait d'utiliser le nombre approximatif de blessures par an et la prévalence des facteurs de risque modifiables (p. ex. l'absence de casque) pour estimer la réduction de la fraction étiologique du risque pour certaines interventions spécifiques. Une telle estimation de l'avantage possible pour la santé publique pourrait servir à déterminer les priorités. Toutefois, cette approche nécessiterait la réalisation d'une méta-analyse fondée sur un sous-ensemble de publications se prêtant à un regroupement des données ou sur l'utilisation de données brutes extraites de la base de données du SCHIRPT. 
TABLEAU 5

Indices de priorité combinés des tableaux 1 à 4

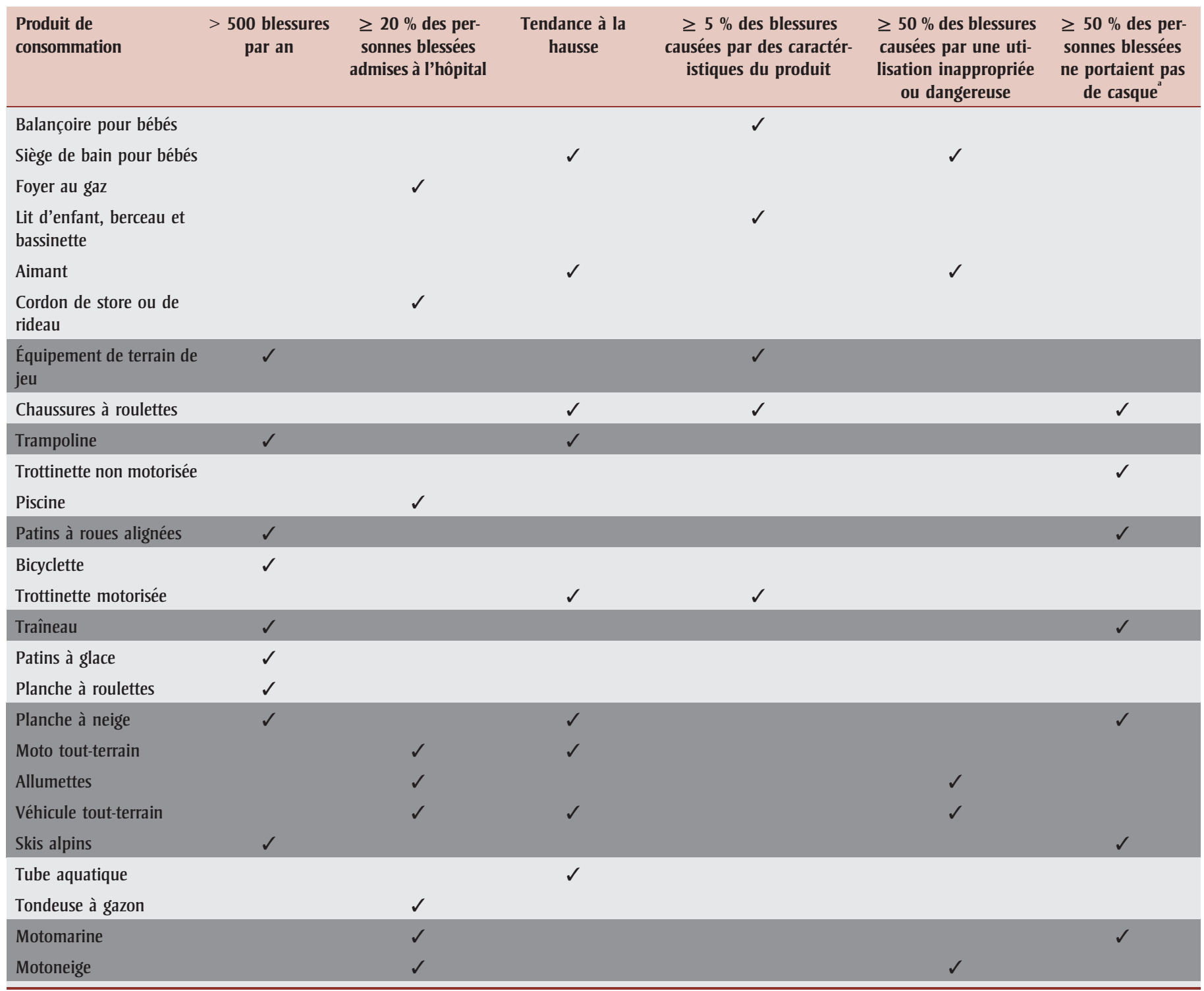

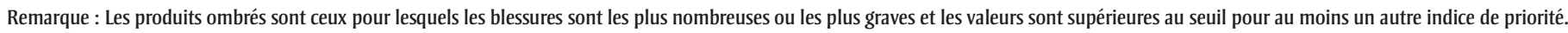

a Lorsque le port du casque est jugé approprié et lorsque cette information a été communiquée.

TABLEAU 6

Produits de consommation et sujets de préoccupation

\begin{tabular}{lc}
\hline Problème & Produit de consommation \\
\hline Absence de casque & Patins à roues alignées \\
& Traîneau \\
& Planche à neige \\
& Skis alpins \\
Utilisation avec les facultés affaiblies & Motomarine \\
Vitesse excessive & Véhicule tout-terrain \\
Conception du produit & Motoneige \\
Entreposage et utilisation inappropriés & Motoneige \\
\hline
\end{tabular}

Estimation du risque fondée sur l'exposition Si cette revue permet de déterminer les types de produits associés au plus grand nombre de blessures, le nombre absolu de blessures est cependant fonction à la fois de la disponibilité d'un produit donné et du risque de blessure qui lui est associé. Pour illustrer cela, demandons-nous si le fait que le nombre de blessures associées aux bicyclettes soit trois à quatre fois plus élevé que celui découlant de l'utilisation de patins à roues alignées indique que le cyclisme est plus dangereux, qu'il y a plus de bicyclettes que de patins à roues alignées ou que les gens passent plus de temps à faire du vélo que du patin à roues 
alignées. Selon toute vraisemblance, la réponse est une combinaison de ces trois facteurs. À quelques exceptions près, les études citées ici ne donnent pas d'estimation du risque de blessures par rapport à l'exposition à un produit, ce qui est une information nécessaire pour repérer les produits particulièrement dangereux et les activités connexes. La difficulté à présenter des estimations comparatives du risque provient du manque de dénominateurs appropriés. À l'avenir, pour au moins certains produits de consommation, on pourrait calculer les taux de blessure en rapport avec l'exposition. On pourrait, afin d'estimer le risque en fonction de l'exposition, utiliser des méthodes similaires à celles utilisées par d'autres auteurs $^{20-23}$ pour établir des liens entre les données du SCHIRPT et celles recueillies dans des enquêtes comme celles sur l'activité physique des jeunes et des adultes obtenues dans le cadre de l'Enquête canadienne sur les mesures de la santé ${ }^{24}$. On pourrait également combiner les données sur les blessures et celles sur les ventes afin de produire une estimation du risque en fonction de la disponibilité de certains produits de consommation.

\section{Taux de blessure dans la population}

Les études citées ici portent sur différents groupes d'âge, différents lieux et différentes périodes, ce qui complique les comparaisons des taux de blessure d'un produit de consommation à l'autre. La base de données du SCHIRPT permet de calculer les taux de blessure par rapport au nombre total de blessures consignées dans la base de données (globalement ou pour un groupe d'âge en particulier); toutefois, ces chiffres ne sont que des approximations des taux dans la population, car, de toute évidence, les personnes répertoriées dans la base de données SCHIRPT ne représentent pas l'ensemble de la population dont elles sont issues. Il serait utile de mettre les données du SCHIRPT en relation avec celles du recensement afin de pouvoir estimer la validité de la première méthode pour estimer les taux (bruts et ajustés) dans la population et d'effectuer des comparaisons entre les produits, ainsi qu'avec d'autres études où des taux dans la population ont été utilisés ${ }^{25,26}$.

\section{Taux par âge}

Si cette revue a permis de déterminer les groupes d'âge les plus touchés par les blessures liées à un produit de consommation donné, elle n'a cependant pas permis de mettre en lumière les produits les plus susceptibles d'être associés à une blessure pour un groupe d'âge donné tout au long de la vie. Les données du SCHIRPT pourraient être utilisées pour fournir cette information, laquelle aiderait à établir les priorités et à élaborer des stratégies de réduction des blessures en relation avec chaque groupe d'âge.

\section{Conclusion}

En dépit des limites citées, cette revue systématique de la littérature offre une vue d'ensemble de la problématique des blessures associées à différents produits de consommation au Canada en termes de fréquence, de gravité, de tendances possibles et de causes probables. Les blessures les plus fréquentes semblent être associées aux équipements de terrain de jeu et aux bicyclettes; les blessures les plus graves surviennent à la maison ou lors de l'utilisation d'un équipement récréatif à moteur; des tendances à la hausse pourraient concerner les blessures associées à plusieurs produits de consommation; enfin, les causes les plus fréquentes des blessures semblent être le comportement de l'utilisateur (c'est-à-dire une utilisation inappropriée ou dangereuse ou des mesures de précaution insuffisantes, par exemple en ce qui concerne le port du casque) plutôt qu'un défaut du produit lui-même. L’analyse présentée ici a permis de répertorier plusieurs sujets de préoccupation concernant les produits de consommation et les blessures : (1) absence de casque chez les utilisateurs de patins à roues alignées, de traîneaux, de planches à neige, de skis alpins et de motomarines; (2) conduite de VTT ou de motoneige avec des facultés affaiblies par l'alcool; (3) conduite de motoneige à une vitesse excessive; (4) conception défectueuse de certains équipements de terrain de jeu; (5) entreposage et utilisation non sécuritaires d'allumettes.

\section{Remerciements}

Les auteurs adressent leurs remerciements à Jane Falconer, bibliothécaire au service d'information de la London School of Hygiene \& Tropical Medicine (RoyaumeUni) pour sa précieuse contribution à l'élaboration de la stratégie de recherche documentaire.

\section{Références}

1. Mo F, Choi BC, Clottey C, LeBrun B, Robbins G. Characteristics and risk factors for accident injury in Canada from 1986 to 1996: an analysis of the Canadian Accident Injury Reporting and Evaluation (CAIRE) database. Inj Control Saf Promot. 2002 Jun;9(2):73-81.

2. Agence de la santé publique du Canada. Système canadien hospitalier d'information et de recherche en prévention des traumatismes (SCHIRPT) [Internet]. Ottawa (Ont.), Agence de la santé publique du Canada; [mise à jour le 22 avr. 2009; consultation le 19 févr. 2012]. Consultable en ligne à la page : http://www.phac-aspc .gc.ca/injury-bles/chirpp/index-fra.php

3. Agence de la Santé publique du Canada. Étude des blessures chez les enfants et les jeunes, édition 2009 - Pleins feux sur la sécurité des produits de consommation. Ottawa (Ont.), Agence de la santé publique du Canada; 2009 [N ${ }^{\circ}$ Cat: HP15-5/2009FPDF au catalogue].

4. Loi canadienne sur la sécurité des produits de consommation, L.C. 2010, ch. 21.

5. Loi sur la sécurité automobile, L.C. 1993, ch. 16.

6. Santé Canada. Équipement de terrains de jeu [Internet]. Ottawa (Ont.), Santé Canada; 2010 [mise à jour le 8 sept. 2010; consultation le 6 déc. 2012]. Consultable en ligne à la page: http://www.hc-sc.gc.ca /cps-spc/sport/equip-play-jeu/index-fra.php

7. World Health Organization. ICD-10 version:2010 [Internet]. Geneva (CH): World Health Organization; 2010 [mise à jour le 4 janv. 2012; consultation le 19 févr. 2012]. Consultable en ligne à la page : http://apps .who.int/classifications/icd10/browse/2010 /en 
8. Agence de la santé publique du Canada. Rapports de blessures [Internet]. Ottawa (Ont.), Agence de la santé publique du Canada; [mise à jour le 30 sept. 2010; consultation le 19 févr. 2012]. Consultable en ligne à la page : http://www.phac-aspc .gc.ca/injury-bles/chirpp/injrep-rapbles /index-fra.php

9. Agence de la santé publique du Canada. Inventaire des sources de données et surveillance des blessures. Ottawa (Ont.), Agence de la santé publique du Canada, Centre de coordination de la surveillance, Direction générale de la santé de la population et de la santé publique; 2005.

10. O’Reilly-Fromentin ME, Rainville M. Portrait des traumatismes liés aux appareils de jeu : données SCHIRPT de l'Hôpital de l'Enfant-Jésus de juillet 1997 à juin 2001. Québec (QC) : Institut national de santé publique du Québec; 2004.

11. Rainville M. Traumatismes d'origine récréative et sportive : portrait des consultations à l'urgence de l'Hôpital de l’Enfant-Jésus de Québec - De juillet 1997 à juin 2001. Québec (QC) : Institut national de santé publique du Québec; 2004.

12. Rainville M, Goulet C, Tremblay B, Maurice P. Blessures en surf des neiges : portrait des consultations à l'urgence de l'Hôpital de l'Enfant-Jésus de Québec entre juin 2003 et décembre 2005. Québec (QC) : Institut national de santé publique du Québec; 2010.

13. Rainville M, Goulet C, Tremblay B, Pierre $\mathrm{M}$. Blessures en planche à roulettes: portrait des consultations à l'urgence de l'Hôpital de l'Enfant-Jésus de Québec entre juin 2003 et décembre 2007. Québec (QC) : Institut national de santé publique du Québec; 2010.

14. Légaré G. Traumatismes associés à l'utilisation des trottinettes au Québec. Bulletin épidémiologique hebdomadaire. 2002;38: 186-187.

15. Montoya FS, Ibarguen AM, Vences AR, Rey ASD, Fernandez JMS. Evaluation of cochlear function in normal-hearing young adults exposed to MP3 player noise by analyzing transient evoked otoacoustic emissions and distortion products. J Otolaryngol Head Neck Surg. 2008;37(5): 718-724.
16. Macarthur C, Pless IB. Evaluation of the quality of an injury surveillance system. Am J Epidemiol. 1999 Mar 15;149(6):586-592.

17. Macarthur C, Pless IB. Sensitivity and representativeness of a childhood injury surveillance system. Inj Prev. 1999 Sep; 5(3):214-216.

18. Macpherson AK, White HL, Mongeon S, Grant VJ, Osmond $\mathrm{M}$, Lipskie $\mathrm{T}$ et al. Examining the sensitivity of an injury surveillance program using populationbased estimates. Inj Prev. 2008 Aug;14(4): 262-265.

19. Macarthur C, Dougherty G, Pless IB. Reliability and validity of proxy respondent information about childhood injury: an assessment of a Canadian surveillance system. Am J Epidemiol. 1997 May 1; 145(9):834-841.

20. Hagel BE, Pless B, Platt RW. Trends in emergency department reported head and neck injuries among skiers and snowboarders. Can J Public Health. 2003 NovDec;94(6):458-462.

21. Aultman-Hall L, Kaltenecker MG. Toronto bicycle commuter safety rates. Accid Anal Prev. 1999 Nov;31(6):675-686.

22. Hu X, Wesson DE, Chipman ML, Parkin PC. Bicycling exposure and severe injuries in school-age children: a population-based study. Arch Pediatr Adolesc Med. 1995; 149(4):437-441.

23. Rowe B, Milner R, Johnson C, Bota G. Snowmobile-related deaths in Ontario: a 5year review. CMAJ. 1992;146(2):147-152.

24. Statistique Canada. Enquête canadienne sur les mesures de la santé : activité physique des jeunes et des adultes [Internet]. Ottawa (Ont.), Gouvernement du Canada; [mise à jour le 5 juill. 2011; consultation le 19 févr. 2012]. Consultable en ligne à la page : http://www.statcan.gc.ca/daily-quotidien /110119/dq110119b-fra.htm

25. Bienefeld M, Pickett W, Carr PA. A descriptive study of childhood injuries in Kingston, Ontario, using data from a computerized injury surveillance system. Chronic Dis Can. 1996;17(1):21-27.
26. Macpherson AK, To TM, Parkin PC, Moldofsky B, Wright JG, Chipman ML et al. Urban/rural variation in children's bicycle-related injuries. Accid Anal Prev. 2004 Jul;36(4):649-654.

27. Agence de la santé publique du Canada. SCHIRPT - Système canadien hospitalier d'information et de recherche en prévention des traumatismes. Échantillon des données : blessures à la tête et au visage causées par la poussette - SCHIRPT, en octobre 2002, chez les enfants de moins de 23 mois - 1751 dossiers [Internet]. Ottawa (Ont.), Agence de la santé publique du Canada; [consultation le 19 févr. 2012]. PDF (76 Ko) téléchargeable à partir du lien : http://www.phac-aspc.gc.ca/injury-bles /chirpp/injrep-rapbles/pdf/strollers_f.pdf

28. Agence de la santé publique du Canada. SCHIRPT Rapports de blessures - Blessures associées aux balançoires pour bébé : recherche exhaustive dans la base de données du SCHIRPT en septembre 1995, chez les enfants moins de 18 mois [Internet]. Ottawa (Ont.), Agence de la santé publique du Canada; [mise à jour le 10 juill. 1996; consultation le 19 févr. 2012]. Consultable en ligne à la page : http://www.phac-aspc.gc.ca/injury-bles /chirpp/injrep-rapbles/irinsw-fra.php

29. Pickett W, Streight S, Simpson K, Brison RJ. Injuries experienced by infant children: a population-based epidemiological analysis. Pediatrics. 2003 Apr;111(4):e365-70.

30. Agence de la santé publique du Canada. SCHIRPT Rapports de blessures - Blessures associées aux marchettes pour bébés, 1990début 2003, chez les enfants de 5 à 14 mois [Internet]. Ottawa (Ont.), Agence de la santé publique du Canada; [consultation le 19 févr. 2012]. PDF (244 Ko) téléchargeable à partir du lien : http://www.phac-aspc .gc.ca/injury-bles/chirpp/pdf/AMU_fr_CHIRPP _INJURY_BRIEF_Baby_Walkers_UPD.pdf

31. Walker JM, Breau L, McNeill D, Rogers B, Sweet K. Hazardous baby walkers: a survey of use. Pediatr Phys Ther. 1996;8(1):25-30. 
32. Agence de la santé publique du Canada. SCHIRPT - Système canadien hospitalier d'information et de recherche en prévention des traumatismes - Échantillon des données : blessures aux barrières pour enfant : SCHIRPT, en octobre 2002, enfants de 0 à 5 ans - 940 dossiers [Internet]. Ottawa (Ont.), Agence de la santé publique du Canada; [consultation le 19 févr. 2012]. PDF (123 Ko) téléchargeable à partir du lien : http://www.phac-aspc.gc.ca/injury-bles /chirpp/injrep-rapbles/pdf/babygates_f.pdf

33. Agence de la santé publique du Canada. SCHIRPT - Système canadien hospitalier d'information et de recherche en prévention des traumatismes - Échantillon des données : blessures aux parcs pour enfant : SCHIRPT, en octobre 2002, chez les enfants de moins de 5 ans - 368 dossiers [Internet]. Ottawa (Ont.), Agence de la santé publique du Canada; [consultation le 19 févr. 2012]. PDF (78 Ko) téléchargeable à partir du lien : http://www.phac-aspc.gc.ca/injury-bles /chirpp/injrep-rapbles/pdf/playpens_f.pdf

34. Zettel JC, Khambalia A, Barden W, Murthy T, Macarthur C. Gas fireplace contact burns in young children. J Burn Care Rehabil. 2004 Nov-Dec;25(6):510-2.

35. Agence de la santé publique du Canada. SCHIRPT Rapports de blessures - Blessures associées aux lits d'enfants, aux berceaux et aux baignoires de bébés : recherche exhaustive dans la base de données du SCHIRPT en février 1996, chez les enfants de moins de 5 ans [Internet]. Ottawa (Ont.), Agence de la santé publique du Canada; [mise à jour le 6 nov. 1997; consultation le 19 févr. 2012]. Consultable en ligne à la page: http://www.phac-aspc.gc.ca/injury-bles /chirpp/injrep-rapbles/crib3-fra.php

36. Agence de la santé publique du Canada. SCHIRPT Rapports de blessures - Blessures associées aux aimants : 1993-2003, 13 ans et moins [Internet]. Ottawa (Ont.), Agence de la santé publique du Canada; [mise à jour le 2 févr. 2012; consultation le 19 févr. 2012]. Consultable en ligne à la page : http://www.phac-aspc.gc.ca/injury-bles /chirpp/injrep-rapbles/magnets-aimant07-fra .php

37. Dotchin SA, Gordon KE. The terrible truth about toppling televisions. Paediatr Child Health. 2007;12(3):221-4.
38. Agence de la santé publique du Canada. Système canadien hospitalier d'information et de recherche en prévention des traumatismes. SCHIRPT Rapport de blessures Blessures associées aux véhicules-jouets motorisés: SCHIRPT, en septembre 2006, chez les enfants de 1 an à 9 ans [Internet]. Ottawa (Ont.), Agence de la santé publique du Canada; [consultation le 19 févr. 2012]. PDF (272 Ko) téléchargeable à partir du lien : http://www.phac-aspc.gc.ca/injury-bles /chirpp/injrep-rapbles/pdf/bmr-vjm-fra.pdf

39. Agence de la santé publique du Canada. SCHIRPT - Système canadien hospitalier d'information et de recherche en prévention des traumatismes - Échantillon des données : blessures attribuables aux lits superposés : SCHIRPT, 1999-2001, 1999 à 2001 - 982 dossiers [Internet]. Ottawa (Ont.), Agence de la santé publique du Canada; [consultation le 19 févr. 2012]. PDF (68 Ko) téléchargeable à partir du lien : http://www.phac-aspc.gc.ca/injury-bles /chirpp/injrep-rapbles/pdf/bunk_bed_fr.pdf

40. Agence de la santé publique du Canada. SCHIRPT - Système canadien hospitalier d'information et de recherche en prévention des traumatismes - Échantillon des données : blessures associées à l'équipement de terrain de jeux : SCHIRPT, sujets de 0 à 14 ans, 2000 - 4225 dossiers [Internet]. Ottawa (Ont.), Agence de la santé publique du Canada; [consultation le 19 févr. 2012]. PDF (63 Ko) téléchargeable à partir du lien : http://www.phac-aspc .gc.ca/injury-bles/chirpp/injrep-rapbles/pdf /playground_f.pdf

41. Fiissel D, Pattison G, Howard A. Severity of playground fractures: play equipment versus standing height falls. Inj Prev. 2005;11(6):337-339.

42. Laforest S, Robitaille Y, Lesage D, Dorval D. Surface characteristics, equipment height, and the occurrence and severity of playground injuries. Inj Prev. 2001 Mar;7(1): $35-40$.

43. Macarthur C, Hu XH, Wesson DE, Parkin PC. Risk factors for severe injuries associated with falls from playground equipment. Accid Anal Prev. 2000;32(3):377-382.

44. Mowat DL, Wang F, Pickett W, Brison RJ. A case-control study of risk factors for playground injuries among children in Kingston and area. Inj Prev. 1998 Mar;4(1):39-43.
45. Agence de la santé publique du Canada. Système canadien hospitalier d'information et de recherche en prévention des traumatismes. Rapport sur les blessures du SCHIRPT - Blessures associées aux chaussures à roulettes (" Heelys ») : SCHIRPT, 2002-2006, tous les âges [Internet]. Ottawa (Ont.), Agence de la santé publique du Canada; [consultation le 19 févr. 2012]. PDF (163 Ko) téléchargeable à partir du lien : http://www.phac-aspc.gc.ca/injury-bles /chirpp/injrep-rapbles/pdf/heely-fra.pdf

46. Agence de la santé publique du Canada. Surveillance des services d'urgence : blessures associées aux dispositifs à roues non motorisés : le Système canadien hospitalier d'information et de recherche en prévention des traumatismes (SCHIRPT), tous les âges, 1990-2007 [Internet]. Ottawa (Ont.), Agence de la santé publique du Canada, Section des blessures et de la violence à l'égard des enfants, Division de la surveillance de la santé et de l'épidémiologie; [consultation le 19 févr. 2012]. PDF (474 Ko) téléchargeable à partir du lien : http: //www.phac-aspc.gc.ca/injury-bles/chirpp /injrep-rapbles/pdf/wheelnonmoto-fra.pdf

47. Thakore S, Tram J, Hagel BE, Kyle T, Senger $\mathrm{T}$, Belanger $\mathrm{F}$. Injuries among wheeled shoe users: a comparison with other nonmotorized wheeled activities. Paediatr Child Health. 2009;14(8):509-513.

48. Agence de la santé publique du Canada. Rapport sur les blessures du SCHIRPT Blessures associées aux trampolines d'extérieur : 1999-2003 (rapport complet) et mise à jour (limitée) de 2004-2006, tous âges [Internet]. Ottawa (Ont.), Agence de la santé publique du Canada; [consultation le 19 févr. 2012]. PDF (154 Ko) téléchargeable à partir du lien : http://www.phac-aspc .gc.ca/injury-bles/chirpp/injrep-rapbles/pdf /trampolines-fra.pdf

49. Black GB, Amadeo R. Orthopedic injuries associated with backyard trampoline use in children. Can J Surg. 2003 Jun;46(3): 199-201. 
50. Agence de la santé publique du Canada. SCHIRPT Rapports de blessures - Blessures associées aux trottinettes sans moteur : base de données du SCHIRPT, jusqu'en mai 2001 : tous âges, juin 2001 (305 déclarations) [Internet]. Ottawa (Ont.), Agence de la santé publique du Canada; [mise à jour le 7 sept. 2001; consultation le 19 févr. 2012]. Consultable en ligne à la page : http: //www.phac-aspc.gc.ca/injury-bles/chirpp /injrep-rapbles/scoot-fra.php

51. Agence de la santé publique du Canada. Système canadien hospitalier d'information et de recherche en prévention des traumatismes. SCHIRPT Rapport de blessures Blessures associées aux noyades et aux quasi-noyades involontaires, 1990-2003, 0 à 14 ans [Internet]. Ottawa (Ont.), Agence de la santé publique du Canada; [consultation le 19 févr. 2012]. PDF (153 Ko) téléchargeable à partir du lien : http: //www.phac-aspc.gc.ca/injury-bles/chirpp /pdf/6925701_003_FR_CHIRPP_INJURY_BRIEF _DROWN.pdf

52. Agence de la santé publique du Canada. Système canadien hospitalier d'information et de recherche en prévention des traumatismes. SCHIRPT Rapport de blessures Parties du corps coincées dans une piscine, une pataugeoire ou un spa, 1990-2003, tous les âges [Internet]. Ottawa (Ont.), Agence de la santé publique du Canada; [consultation le 19 févr. 2012]. PDF (238 Ko) téléchargeable à partir du lien : http: //www.phac-aspc.gc.ca/injury-bles/chirpp /pdf/6925701_006_FR_CHIRPP_INJURY_BRIEF _POOL_ENTRAP.pdf

53. Agence de la santé publique du Canada. Système canadien hospitalier d'information et de recherche en prévention des traumatismes. SCHIRPT Rapport de blessures Blessures associées aux feux d'artifice, aux pétards et aux feux de Bengale : cumul de juin 1998 à mars 2004 [Internet]. Ottawa (Ont.), Agence de la santé publique du Canada; [consultation le 19 févr. 2012]. PDF (147 Ko) téléchargeable à partir du lien : http://www.phac-aspc.gc.ca/injury-bles /chirpp/pdf/CHIRPP_INJURY_BRIEF_Fireworks _FR.pdf
54. Agence de la santé publique du Canada. SCHIRPT Rapport de blessures - Blessures associées aux patins à roues alignées : base de données du SCHIRPT, données sommaires pour 1998, tous âges [Internet]. Ottawa (Ont.), Agence de la santé publique du Canada; [consultation le 19 févr. 2012]. Consultable en ligne à la page : http: //www.phac-aspc.gc.ca/injury-bles/chirpp /injrep-rapbles/inline-fra.php

55. Agence de la santé publique du Canada. Système canadien hospitalier d'information et de recherche en prévention des traumatismes. SCHIRPT Rapport de blessures Blessures associées aux bicyclettes, 2006, 1 an et plus [Internet]. Ottawa (Ont.), Agence de la santé publique du Canada; [consultation le 19 févr. 2012]. PDF (59 Ko) téléchargeable à partir du lien : http: //www.phac-aspc.gc.ca/injury-bles/chirpp /pdf/chirpp-bikes-nov2008-fra.pdf

56. Rowe BH, Rowe AM, Bota GW. Bicyclist and environmental factors associated with fatal bicycle-related trauma in Ontario. CMAJ. 1995;152(1):45-53.

57. Yanchar NL, Kennedy R, Russell C. ATVs: motorized toys or vehicles for children? Inj Prev. 2006 Feb;12(1):30-34.

58. Agence de la santé publique du Canada. Système canadien hospitalier d'information et de recherche en prévention des traumatismes. SCHIRPT Rapport de blessures Blessures associées aux go-karts: SCHIRPT, 2000-2002, chez les enfants âgés de 5 ans et plus [Internet]. Ottawa (Ont.), Agence de la santé publique du Canada; [consultation le 19 févr. 2012]. PDF (318 Ko) téléchargeable à partir du lien : http://www.phac-aspc .gc.ca/injury-bles/chirpp/injrep-rapbles/pdf /gc-gk-fra.pdf

59. Agence de la santé publique du Canada. Surveillance du service des urgences Blessures associées aux véhicules récréatifs motorisés hors route, Système canadien hospitalier d'information et de recherche en prévention des traumatismes (SCHIRPT), tous âges, 1990-2007 [Internet]. Ottawa (Ont.), Agence de la santé publique du Canada; [consultation le 19 févr. 2012]. PDF (475 Ko) téléchargeable à partir du lien : http://www.phac-aspc.gc.ca/injury-bles /chirpp/injrep-rapbles/pdf/highway-fra.pdf
60. Agence de la santé publique du Canada. Système canadien hospitalier d'information et de recherche en prévention des traumatismes. SCHIRPT Rapport de blessures Blessures associées aux trottinettes motorisées: SCHIRPT, 1998-2005, groupe d'âge 7 ans et plus [Internet]. Ottawa (Ont.), Agence de la santé publique du Canada; [consultation le 19 févr. 2012]. PDF (315 Ko) téléchargeable à partir du lien : http: //www.phac-aspc.gc.ca/injury-bles/chirpp /pdf/6925701_007_FR_CHIRPP_INJURY _BRIEF_POWERED_SCOOTER.pdf

61. Agence de la santé publique du Canada. Système canadien hospitalier d'information et de recherche en prévention des traumatismes. SCHIRPT Rapport de blessures Blessures associées aux activités et aux sports de glace ou de neige, 2000-2002, groupe d'âge - cinq ans ou plus [Internet]. Ottawa (Ont.), Agence de la santé publique du Canada; [consultation le 19 févr. 2012]. PDF (191 Ko) téléchargeable à partir du lien : http://www.phac-aspc.gc.ca/injury-bles /chirpp/injrep-rapbles/pdf/is-gn-fra.pdf

62. Agence de la santé publique du Canada. Système canadien hospitalier d'information et de recherche en prévention des traumatismes. SCHIRPT Rapport de blessures Blessures associées à la glissade, hiver 2001-2002 [Internet]. Ottawa (Ont.), Agence de la santé publique du Canada; [mise à jour le 22 avr. 2009; consultation le 19 févr. 2012]. PDF (101 Ko) téléchargeable à partir du lien : http://www.phac-aspc .gc.ca/injury-bles/chirpp/injrep-rapbles/pdf /sledding-glissade01-02-fra.pdf

63. Voaklander DC, Kelly KD, Sukrani N, Sher A, Rowe BH. Sledding injuries in patients presenting to the emergency department in a northern city. Acad Emerg Med. 2001 Jun;8(6):629-35.

64. Wynne AD, Bota GW, Rowe BH. Sledding trauma in a northeastern Ontario community. J Trauma. 1994;37(5):820-5. 
65. Agence de la santé publique du Canada. SCHIRPT - Système canadien hospitalier d'information et de recherche en prévention des traumatismes - Échantillon de données : blessures associées aux sports sur neige [Internet]. Ottawa (Ont.), Agence de la santé publique du Canada; [consultation le 19 févr. 2012]. PDF (31 Ko) téléchargeable à partir du lien : http://www .phac-aspc.gc.ca/injury-bles/chirpp/injrep -rapbles/pdf/snow-sport_sports-neige07_f .pdf

66. Agence de la santé publique du Canada. Système canadien hospitalier d'information et de recherche en prévention des traumatismes. SCHIRPT Rapport de blessures Blessures associées aux allumettes 19932003, groupe d'âge 15 ans ou moins [Internet]. Ottawa (Ont.), Agence de la santé publique du Canada; [mise à jour le 8 déc. 2008; consultation le 19 févr. 2012]. Consultable en ligne à la page : http: //www.phac-aspc.gc.ca/injury-bles/chirpp /chirpp-matches-nov2008-fra.php

67. Agence de la santé publique du Canada. SCHIRPT - Système canadien hospitalier d'information et de recherche en prévention des traumatismes - Échantillon de données : blessures associées aux véhicules tout terrain (VTT) : SCHIRPT, 1999-2001, tous âges- 895 dossiers. [Internet]. Ottawa (Ont.), Agence de la santé publique du Canada; [consultation le 19 févr. 2012]. PDF (77 Ko) téléchargeable à partir du lien : http://www.phac-aspc.gc.ca/injury-bles /chirpp/injrep-rapbles/pdf/atv_f.pdf

68. Alawi K, Lynch T, Lim R. All-terrain vehicle major injury patterns in children: a fiveyear review in Southwestern Ontario. CJEM. 2006 Jul;8(4):277-280.

69. Lim GW, Belton KL, Pickett W, Schopflocher DP, Voaklander DC. Fatal and non-fatal machine-related injuries suffered by children in Alberta, Canada, 19901997. Am J Ind Med. 2004 Feb;45(2): 177-185.

70. Murphy N, Yanchar NL. Yet more pediatric injuries associated with all-terrain vehicles: should kids be using them? J Trauma. 2004;56(6):1185-1190.

71. Sibley AK, Tallon JM. Major injury associated with all-terrain vehicle use in Nova Scotia: a 5-year review. CJEM. 2002;4(4): 263-267.
72. Su W, Hui T, Shaw K. All-terrain vehicle injury patterns: are current regulations effective? J Pediatr Surg. 2006;41(5):931934.

73. Macnab AJ, Cadman R. Demographics of alpine skiing and snowboarding injury: lessons for prevention programs. Inj Prev. 1996 Dec;2(4):286-289.

74. Agence de la santé publique du Canada. Surveillance des services d'urgence : blessures associées au tube aquatique et au ski nautique, Le Système canadien hospitalier d'information et de recherche en prévention des traumatismes (SCHIRPT), tous les âges, 1990-2008 [Internet]. Ottawa (Ont.), Agence de la santé publique du Canada, Section des blessures et de la violence envers les enfants, Division de la surveillance de la santé et de l'épidémiologie; [consultation le 19 févr. 2012]. PDF (575 Ko) téléchargeable à partir du lien : http: //www.phac-aspc.gc.ca/injury-bles/chirpp /pdf/wtski-fra.pdf

75. Agence de la santé publique du Canada. Système canadien hospitalier d'information et de recherche en prévention des traumatismes. SCHIRPT Rapport de blessures Blessures associées aux tondeuses à gazon, 1990-2006, tous les groupes d'âge [Internet]. Ottawa (Ont.), Agence de la santé publique du Canada; [consultation le 19 févr. 2012]. PDF (129 Ko) téléchargeable à partir du lien : http://www.phac-aspc .gc.ca/injury-bles/chirpp/pdf/7164973_002_FR _CHIRPP_INJURY_BRIEF_LAWNMOWERS .pdf

76. Agence de la santé publique du Canada. SCHIRPT Rapport de blessures - Blessures associées aux motos marines : base de données du SCHIRPT, à partir de juin 1998, tous âges [Internet]. Ottawa (Ont.), Agence de la santé publique du Canada; [mise à jour le 11 janv. 1999; consultation le 19 févr. 2012]. Consultable en ligne à la page : http://www.phac-aspc.gc.ca/injury-bles /chirpp/injrep-rapbles/jetski-fra.php

77. Stewart RL, Black GB. Snowmobile trauma: 10 years' experience at Manitoba's tertiary trauma centre. Can J Surg. 2004 Apr;47(2): 90-94.
78. Kirby RL, Ackroyd-Stolarz SA, Brown MG, Kirkland SA, MacLeod DA. Wheelchairrelated accidents caused by tips and falls among noninstitutionalized users of manually propelled wheelchairs in Nova Scotia. Am J Phys Med Rehabil. 1994;73(5): 319-330.

79. Sherman GJ. Trampoline-related injuries in the Children's Hospitals Injury, Research and Prevention Program. Chronic Dis Can. 1991;12(5):78-80.

80. Hagel BE, Russell K, Goulet C, NettelAguirre A, Pless IB. Helmet use and risk of neck injury in skiers and snowboarders. Am J Epidemiol. 2010 May 15;171(10): 1134-1143. 